\title{
Autoimmunity plays a role in the onset of diabetes after 40 years of age
}

\author{
Olov Rolandsson ${ }^{1}$ (1) $\cdot$ Christiane S. Hampe ${ }^{2}$ Stephen J. Sharp ${ }^{3} \cdot$ Eva Ardanaz $^{4,5,6} \cdot$ Heiner Boeing $^{7} \cdot$ Guy Fagherazzi $^{8}$. \\ Francesca Romana Mancini ${ }^{8} \cdot$ Peter M. Nilsson ${ }^{9} \cdot$ Kim Overvad $^{10,11} \cdot$ Maria-Dolores Chirlaque $^{5,12}$. \\ Miren Dorronsoro ${ }^{5,13,14}$. Marc J. Gunter ${ }^{15}$. Rudolf Kaaks ${ }^{16} \cdot$ Timothy J. Key $^{17} \cdot$ Kay-Tee Khaw ${ }^{18} \cdot$ Vittorio Krogh $^{19}$. \\ Tilman Kühn ${ }^{16}$. Domenico Palli ${ }^{20}$. Salvatore Panico ${ }^{21}$ • Carlotta Sacerdote ${ }^{22}$ - Maria-José Sánchez ${ }^{5,23,24}$. \\ Gianluca Severi $^{25,26}$. Annemieke M. W. Spijkerman ${ }^{27}$ • Rosario Tumino ${ }^{28,29}$ • Yvonne T. van der Schouw ${ }^{30}$. \\ Elio Riboli ${ }^{31}$ • Nita G. Forouhi ${ }^{3} \cdot$ Claudia Langenberg $^{3} \cdot$ Nicholas J. Wareham $^{3}$
}

Received: 17 December 2018 / Accepted: 22 August 2019 / Published online: 11 November 2019

(C) The Author(s) 2019

\begin{abstract}
Aims/hypothesis Type 1 and type 2 diabetes differ with respect to pathophysiological factors such as beta cell function, insulin resistance and phenotypic appearance, but there may be overlap between the two forms of diabetes. However, there are relatively few prospective studies that have characterised the relationship between autoimmunity and incident diabetes. We investigated associations of antibodies against the $65 \mathrm{kDa}$ isoform of GAD (GAD65) with type 1 diabetes and type 2 diabetes genetic risk scores and incident diabetes in adults in European Prospective Investigation into Cancer and Nutrition (EPIC)-InterAct, a casecohort study nested in the EPIC cohort.

Methods GAD65 antibodies were analysed in EPIC participants (over 40 years of age and free of known diabetes at baseline) by radioligand binding assay in a random subcohort $(n=15,802)$ and in incident diabetes cases $(n=11,981)$. Type 1 diabetes and type 2 diabetes genetic risk scores were calculated. Associations between GAD65 antibodies and incident diabetes were estimated using Prentice-weighted Cox regression.

Results GAD65 antibody positivity at baseline was associated with development of diabetes during a median follow-up time of 10.9 years (HR for GAD65 antibody positive vs negative 1.78; 95\% CI 1.43, 2.20) after adjustment for sex, centre, physical activity, smoking status and education. The genetic risk score for type 1 diabetes but not type 2 diabetes was associated with GAD65 antibody positivity in both the subcohort (OR per SD genetic risk 1.24; 95\% CI 1.03, 1.50) and incident cases (OR 1.97; 95\% CI 1.72, 2.26) after adjusting for age and sex. The risk of incident diabetes in those in the top tertile of the type 1 diabetes genetic risk score who were also GAD65 antibody positive was 3.23 (95\% CI 2.10 , 4.97) compared with all other individuals, suggesting that $1.8 \%$ of incident diabetes in adults was attributable to this combination of risk factors.

Conclusions/interpretation Our study indicates that incident diabetes in adults has an element of autoimmune aetiology. Thus, there might be a reason to re-evaluate the present subclassification of diabetes in adulthood.
\end{abstract}

Keywords Autoantibody $\cdot$ Autoimmunity $\cdot$ Genetic risk score $\cdot$ Incident diabetes $\cdot$ Type 1 diabetes $\cdot$ Type 2 diabetes

Electronic supplementary material The online version of this article (https://doi.org/10.1007/s00125-019-05016-3) contains peer-reviwed but unedited supplementary material, which is available to authorised users.

Olov Rolandsson

olov.rolandsson@umu.se

Extended author information available on the last page of the article

$\begin{array}{ll}\text { Abbreviations } \\ \text { EPIC } & \begin{array}{l}\text { European Prospective Investigation into Cancer } \\ \text { and Nutrition }\end{array} \\ \text { GAD65 } & \text { 65 kDa isoform of GAD } \\ \text { GRS } & \text { Genetic risk score } \\ \text { HUNT } & \text { The Nord-Trøndelag Health Study } \\ \text { rhGAD65 } & \text { Recombinant human GAD65 } \\ \text { ROC } & \text { Receiver operating characteristic }\end{array}$

Abbreviations and Nutrition

Genetic risk score

Recombinant human GAD65

ROC Receiver operating characteristic 


\section{Research in context}

\section{What is already known about this subject?}

- Type 1 diabetes has an autoimmune aetiology

- Type 2 diabetes is associated with insulin resistance and insulin secretory deficit

- There may be an overlap between the two subtypes

\section{What is the key question?}

- Is autoimmunity, assessed by presence of antibodies against GAD65, associated with incident adult-onset diabetes and do genetic risk scores for type 1 diabetes or type 2 diabetes modify the association?

\section{What are the new findings?}

- GAD65 antibody positivity at baseline in people who were over 40 years of age and free of known diabetes was associated with development of diabetes during a median follow-up time of 10.9 years (HR for GAD65 antibody positive vs negative $1.78,95 \% \mathrm{Cl} 1.43,2.20$ )

- The genetic risk score for type 1 diabetes but not type 2 diabetes was associated with GAD65 antibody positivity in both a random subcohort of EPIC-InterAct and among incident cases of diabetes

- Among GAD65 antibody-positive individuals there was a significant association of the type 1 diabetes genetic risk score with incident diabetes

\section{How might this impact on clinical practice in the foreseeable future?}

- Our study indicates that incident diabetes in adults has an element of autoimmune aetiology and that there may, therefore, be a reason to re-evaluate the present subclassification of diabetes in adulthood

\section{Introduction}

Diabetes mellitus has classically been thought to have two major aetiological subtypes: type 1 diabetes, which is characterised by autoimmune destruction of beta cells with subsequent insulin deficiency, and type 2 diabetes, which is associated with insulin resistance and a relative insulin secretory deficit [1]. In type 1 diabetes the autoimmune response manifests itself in T cell reactivity and autoantibody responses directed against at least four beta cell autoantigens, including the $65 \mathrm{kDa}$ isoform of GAD (GAD65) [2]. Although the pathogenesis of type 2 diabetes is thought to be different from that of type 1 diabetes, there may be some overlap; however, the evidence for this is limited [3]. Associations of beta cell autoimmunity, as assessed by presence of GAD65 antibodies, with insulin requirement and diabetes complications have been investigated in prevalent adult-onset diabetes [4-14]. In contrast, there are only a few population-based prospective studies exploring the association between autoimmunity and incident diabetes. Moreover, while some of these studies reported an association $[15,16]$, others have been inconclusive [17-19]. In a recent meta-analysis Koopman et al reported that the pooled risk estimate of incident type 2 diabetes for GAD65 antibody positivity, compared with GAD65 antibody negativity, was 3.36 (95\% CI $1.9,5.9)$ [20]. However, there was significant heterogeneity between the studies in the meta-analysis.
Susceptibility for development of GAD65 antibody and type 1 diabetes is at least in part explained by risk alleles located within the HLA region on chromosome 6 [21-23]. We have previously shown that specific HLA haplotypes are associated with GAD65 antibody positivity [23]. It is unknown whether a possible association between GAD65 antibodies and adult-onset diabetes is explained by these HLA haplotypes. It has also been suggested that type 2 diabetes risk alleles are associated with a subset of adult-onset diabetes characterised by presence of GAD65 antibodies at or around the time of diagnosis [24-26], but this association may be affected by the level of the cut-off used to define presence of autoantibodies [27].

Thus, we aimed to investigate the association between GAD65 antibody levels measured using a competition assay, which increases the precision of the assessment of autoantibody positivity, and incident adult-onset diabetes in a large, multi-centre, population-based prospective study in eight European countries in people who were over 40 years of age and free of known diabetes at baseline. In addition, we investigated whether genetic risk scores (GRSs) for type 1 diabetes and type 2 diabetes are associated with GAD65 antibody positivity, and the potential for either of these risk scores to modify the association of GAD65 antibodies with incident adultonset diabetes. 


\section{Methods}

Population The design and methods of the European Prospective Investigation into Cancer and Nutrition (EPIC)InterAct case-cohort study have previously been described [28]. A total of 340,234 EPIC participants, who were over the age of 40 and free of known diabetes at baseline, in eight of the ten EPIC study countries ( 26 centres) were followed up for 3.99 million person-years (median follow-up 10.9 years), during which 12,403 incident cases of diabetes were ascertained and verified. The mean (SD) age at diagnosis was $62.3(7.8)$ years in men and 62.6 (8.2) years in women [28]. Ascertainment of incident diabetes involved multiple sources of evidence including self-report, linkage to primary care registers, secondary care registers, medication use (drug registers), hospital admissions and mortality data, with a minimum of two data sources being required to confirm the diagnosis. Cases in Denmark and Sweden were not ascertained by self-report but identified via local and national diabetes and pharmaceutical registers, and hence all ascertained cases were considered to be verified. Information from any follow-up visit or external evidence with a date later than the baseline visit was used. Follow-up was censored at the date of diagnosis, 31 December 2007 or the date of death, whichever occurred first. A centre-stratified subcohort of 16,835 (4.9\% of the entire EPIC cohort) individuals was selected at random [28]. We excluded 548 individuals with known prevalent diabetes and 133 with unknown diabetes status at baseline. We also excluded 422 cases and 352 subcohort participants (of whom 34 were incident cases) with insufficient sample volume for GAD65 antibody measurement, resulting in 15,802 subcohort participants and 11,981 incident cases being included in the analysis.

All study participants gave informed consent, and the investigation has been carried out in accordance with the Declaration of Helsinki as revised in 2008.

GAD65 antibody measurement Blood samples were drawn at the time of participation in EPIC, at which time all participants were free of known diabetes. Blood plasma was prepared and stored at $-196^{\circ} \mathrm{C}$ in liquid nitrogen at the coordinating centre at the International Agency for Research into Cancer (IARC) in Lyon, France, or in liquid nitrogen in local biorepositories except for Umeå, where $-80^{\circ} \mathrm{C}$ freezers were used [28].

The samples had been subject to at least two freeze-thaw cycles before being analysed for GAD65 antibody. Recombinant ${ }^{[35]} \mathrm{S}-$ GAD65 was produced in an in vitro coupled transcription and translation system with SP6 RNA polymerase and nuclease-treated rabbit reticulocyte lysate (Promega, Madison, WI, USA) as previously described [29]. The WHO standard [30] was included and used to express immunoglobulin binding levels in relative units.

To determine the cut-off for GAD65 antibody positivity, we used a competition assay employing recombinant human
GAD65 (rhGAD65) (Diamyd Medical, Stockholm, Sweden) as previously described [31]. A total of 900 serum samples were randomly selected across countries in the EPIC-InterAct study population. The samples were incubated with radiolabelled GAD65 in the absence or presence of rhGAD65 (200 ng/ml) or BSA (200 ng/ml). Samples in which binding to radiolabelled GAD65 was reduced by $50 \%$ in the presence of rhGAD65, but not BSA, were considered to be positive for GAD65-specific antibodies. We used 50\% as a cut-off for successful competition as an approximation of the $\mathrm{IC}_{50}$. Given the low sample volume, we chose to use the competitor at the optimal concentration $(200 \mathrm{ng} / \mathrm{ml})$ found in previous experiments, in which we titrated the amount of rhGAD65 necessary to give maximal competition. In a variation of the traditional receiver operating characteristic (ROC) analysis, we plotted GAD65 antibody levels of samples that were competed by $\geq 50 \%$ against GAD65 antibody levels of samples that were competed by $<50 \%$. The area under the ROC curve was 0.97 , indicating excellent predictive ability of the GAD65 antibody measurement. At a cut-off level of $\geq 65 \mathrm{U} / \mathrm{ml}$, the measurement had $99 \%$ specificity and $85 \%$ sensitivity (electronic supplementary material [ESM] Fig. 1). Thereafter, all samples in the subcohort $(n=15,802)$ and incident cases $(n=11,981)$ were analysed for GAD65 antibodies in a radiobinding assay (RBA) as previously described [29].

Measurement of covariates Weight, height, and waist and hip circumferences were measured with participants not wearing shoes and in light clothing or underwear, as described previously [28]. BMI was calculated as weight/height squared $(\mathrm{kg} /$ $\mathrm{m}^{2}$ ). Waist circumference was measured either at the narrowest circumference of the torso or at the midpoint between the lower ribs and the iliac crest. Hip circumference was measured horizontally at the level of the largest lateral extension of the hips or over the buttocks. Anthropometric data were mostly self-reported in the Oxford centre, and waist and hip circumferences were not measured in the Umeå centre $(n=1845)$.

Standardised information on highest educational level (none, primary, technical, secondary or further education) and smoking status (current smoker, never a smoker or former smoker) was collected by questionnaire at baseline [28]. Physical activity was assessed using a brief questionnaire covering occupation and recreational activity, from which a validated physical activity index (inactive, moderately inactive, moderately active or active) was derived [32].

Genetic analysis and GRS Samples were processed for arraybased genotyping if they had sufficient DNA that could be successfully genotyped on TaqMan (Thermo Fisher Scientific, Waltham, MA, USA) or Sequenom (San Diego, CA, USA) platforms and had sex chromosome genotypes concordant with self-reported sex. Samples that failed one 
genotyping round for reasons that did not relate to sample quality (e.g. signal intensity outliers or plates/arrays with an unusually high failure rate) were repeated. Samples were genotyped on the Illumina 660 W-Quad BeadChip, the Illumina HumanCoreExome-12 or the Illumina HumanCoreExome-24 (San Diego, CA, USA). Samples genotyped on the Illumina $660 \mathrm{~W}$-Quad BeadChip were randomly selected from the available samples with the number of individuals selected per centre being proportional to the percentage of total cases in that centre. The Danish samples were not available for genotyping at this stage. Genotyping was carried out at the Wellcome Trust Sanger Institute. Most of the remaining non-Danish samples were genotyped on the Illumina HumanCoreExome-12 at Cambridge Genomic Services in the University of Cambridge Department of Pathology. Finally, the Danish samples and repeat samples due to poor genotyping were genotyped on the Illumina HumanCoreExome-24 also at Cambridge Genomic Services. Sample quality control criteria varied slightly by array but included call rate $(<95.4 \%$ in Illumina $660,<98 \%$ in core exome arrays), $\mathrm{X}$ chromosome heterozygosity concordance with self-reported sex, outliers for heterozygosity and concordance with previous genotyping results.

From the genome-wide array data, we calculated a type 1 diabetes GRS as a weighted average of 33 SNPs, including five HLA variants. The relevant SNPs and their individual associations with GAD65 antibody positivity are described in ESM Table 1 [33]. We also calculated a type 2 diabetes GRS as a weighted average of 68 SNPs from a DIAbetes Genetics Replication And Meta-analysis (DIAGRAM) consortium publication [34].

Statistical analyses Baseline characteristics of the analysis sample were summarised by GAD65 antibody status (negative/positive), separately within the subcohort and incident diabetes cases, using means and standard deviations for continuous variables (except for GAD65 antibody level, which had a skewed distribution so the median and interquartile range were used) and percentages for categorical variables.

The association between GAD65 antibody status (positive/ negative) and incident diabetes was estimated using Prenticeweighted Cox regression, which is appropriate for estimating association in a case-cohort study. We fitted models within each country and the estimated HRs were combined across countries using random effects meta-analysis. We fitted three models including the following covariates: Model 1-age (as underlying time scale), sex and centre; Model 2-also including physical activity, smoking status and education; Model 3 -also including family history of diabetes. In order to study the effects of high vs low GAD65 antibody levels, GAD65 antibody-positive individuals were further subdivided into those with GAD65 antibody equal to or above, and those with GAD65 antibody below, $167.5 \mathrm{U} / \mathrm{ml}$, which is the median antibody level in the GAD65 antibody-positive group in this study. Prentice-weighted Cox regression was also used to test possible multiplicative interactions of GAD65 antibody status with: (1) sex; (2) BMI category; (3) waist/hip ratio (WHR) category (sex-specific tertiles); and (4) type 1 diabetes GRS tertile. The interactions with anthropometry measures were tested because of prior studies suggesting that adiposity could moderate the association of autoimmunity with diabetes $[9,10]$. In this instance we fitted models to the overall dataset with adjustment for country, due to insufficient data within each country to obtain country-specific estimates. HRs and 95\% CIs within each subgroup were calculated.

The associations of the type 1 diabetes GRS and the type 2 diabetes GRS with GAD65 antibody status (positive/negative) were estimated separately in the subcohort and the incident diabetes cases, using logistic regression adjusted for age and sex, since the prevalence of autoimmunity is associated with age and sex. Models were fitted within each country and estimated odds ratios combined across countries using random effects meta-analysis. ORs (and 95\% CIs) per risk allele of each of the individual SNPs contributing to the type 1 diabetes GRS were also calculated in the subcohort using the same method.

The association between the type 1 diabetes GRS and incident diabetes, by GAD65 antibody status, was estimated using Prentice-weighted Cox regression as described above.

\section{Results}

Three hundred and sixteen (2.0\%) individuals in the subcohort and $413(3.4 \%)$ incident cases were GAD65 antibody positive as defined by having a level of $\geq 65 \mathrm{U} / \mathrm{ml}$ (Table 1 ). Individuals who were GAD65 antibody positive in both the subcohort and incident cases tended to be leaner, and fewer of these individuals reported a family history of diabetes. There were no other major differences in baseline characteristics by GAD65 antibody status (Table 1). The distributions of the type 1 diabetes GRS and the type 2 diabetes GRS were similar when comparing individuals who were GAD65 antibody positive and negative, in both the subcohort and incident diabetes cases (Table 1, Fig. 1a,b).

GAD65 antibody positivity was associated with a higher incidence of diabetes (Table 2) consistently across countries (Fig. 2). Of the potential confounders considered, only family history of diabetes had an impact on the HR, which increased from $1.78(95 \%$ CI 1.43, 2.20) in Model 2 to 2.19 (95\% CI $1.56,3.07)$ in Model 3, which includes family history of diabetes. Data on family history of diabetes were not collected at some centres; fitting all three models to the sample with complete data on all covariates gave results similar to those above (ESM Table 2), suggesting the presence of negative confounding by family history. The association was markedly 
Table 1 Baseline characteristics of the subcohort and incident diabetes cases; the EPIC-InterAct study $(N=27,039)$

\begin{tabular}{|c|c|c|c|c|}
\hline \multirow[t]{2}{*}{ Characteristic } & \multicolumn{2}{|l|}{ Subcohort } & \multicolumn{2}{|l|}{ Incident diabetes cases } \\
\hline & GAD65 antibody ${ }^{-} n=15,486$ & GAD65 antibody $^{+} n=316$ & GAD65 antibody $^{-} n=11,568$ & GAD65 antibody ${ }^{+} n=413$ \\
\hline Women $(\%)$ & 62.4 & 64.6 & 50.2 & 58.1 \\
\hline Age (years) & $52.3(9.1)$ & $52.7(9.3)$ & $55.5(7.6)$ & $55.0(8.4)$ \\
\hline BMI $\left(\mathrm{kg} / \mathrm{m}^{2}\right)$ & $26.0(4.2)$ & $25.8(4.2)$ & $29.8(4.7)$ & $28.4(5.1)$ \\
\hline WHR & $0.85(0.09)$ & $0.85(0.09)$ & $0.92(0.09)$ & $0.89(0.09)$ \\
\hline \multicolumn{5}{|l|}{ Physical activity } \\
\hline Inactive & 23.7 & 25.2 & 30.1 & 33.3 \\
\hline Moderately inactive & 33.5 & 35.8 & 32.9 & 28.0 \\
\hline Moderately active & 22.7 & 19.5 & 20.3 & 20.2 \\
\hline Active & 20.1 & 19.5 & 16.7 & 18.5 \\
\hline \multicolumn{5}{|l|}{ Highest schooling level } \\
\hline None & 7.7 & 10.3 & 10.1 & 8.6 \\
\hline Primary & 33.0 & 34.3 & 41.9 & 40.9 \\
\hline Technical & 23.3 & 20.8 & 23.6 & 21.9 \\
\hline Secondary & 15.4 & 14.7 & 11.1 & 14.5 \\
\hline Further education & 20.7 & 19.9 & 13.3 & 14.0 \\
\hline \multicolumn{5}{|l|}{ Smoking status } \\
\hline Never & 47.0 & 50.5 & 41.0 & 43.2 \\
\hline Former & 27.1 & 25.2 & 31.2 & 28.0 \\
\hline Current & 25.9 & 24.3 & 27.8 & 28.8 \\
\hline Family history of diabetes (yes) & 18.7 & 14.5 & 36.3 & 29.5 \\
\hline GAD65 antibody (U/ml) & $0.0(0,0)$ & $133.3(86.7,336.1)$ & $0.0(0,0)$ & $207.1(99.3,1000)$ \\
\hline Type 1 diabetes risk score & $0.6(0.1)$ & $0.6(0.1)$ & $0.6(0.1)$ & $0.6(0.1)$ \\
\hline Type 2 diabetes risk score & $70.6(5.7)$ & $70.4(5.2)$ & $72.4(5.7)$ & $71.6(5.7)$ \\
\hline
\end{tabular}

Values are presented as percentages for categorical variables and mean (SD) for continuous variables, except for GAD65 antibody, which has a skewed distribution and so median (interquartile range) is presented

stronger in those with high GAD65 antibody levels than in those with lower levels, relative to those who were GAD65 antibody negative (Table 2), suggesting a potential doseresponse effect. We undertook a sensitivity analysis to examine the effect of lowering the threshold for defining GAD65 antibody positivity from $\geq 65 \mathrm{U} / \mathrm{ml}$ to $\geq 40 \mathrm{U} / \mathrm{ml}$. This would lead to an additional 255 incident diabetes cases being labelled as GAD65 antibody positive, but led to a weaker overall association between GAD65 antibody positivity and incident diabetes. A formal comparison of the effect size for the association with incident diabetes for the two definitions of GAD65 antibody positivity was not significant $(p=0.099)$.

Type 1 diabetes GRS, but not type 2 diabetes GRS, was associated with GAD65 antibody positivity in both the subcohort and incident diabetes cases (Table 3). Of the five SNPs in the type 1 diabetes GRS that are linked to HLA haplotypes, three were significantly associated with GAD65 antibody positivity in the subcohort after adjusting for age and sex (ESM Table 2). However, HLA haplotypes did not explain the association between GAD65 antibodies and incident diabetes. In a sensitivity analysis restricted to those individuals with complete relevant data $(n=10,893)$, the HR for incident diabetes in those positive for GAD65 antibodies compared with those individuals who were negative was 2.22 (95\% CI 1.49, 3.31 ) without adjustment and 2.13 (95\% CI 1.46, 3.11) with adjustment for a five HLA haplotype risk score. There was no association between any individual type 2 diabetes-associated SNP and GAD65 antibody level (ESM Table 3).

There was no overall association between type 1 diabetes GRS and incident diabetes (HR 1.02 per SD type 1 diabetes GRS; 95\% CI 0.99, 1.06) when adjusted for age, sex, physical activity, smoking status, education and BMI. However, there were significant interactions of GAD65 antibody status with BMI category $(p=0.001)$, WHR category $(p<0.001)$ and type 1 diabetes GRS tertile $(p<0.001)$, but no interaction of GAD65 antibody status with sex, on the hazard of diabetes. The associations between GAD65 antibody positivity and incident diabetes were strongest in the lowest categories of BMI and WHR, and in the highest tertile of type 1 diabetes GRS (Table 4). 


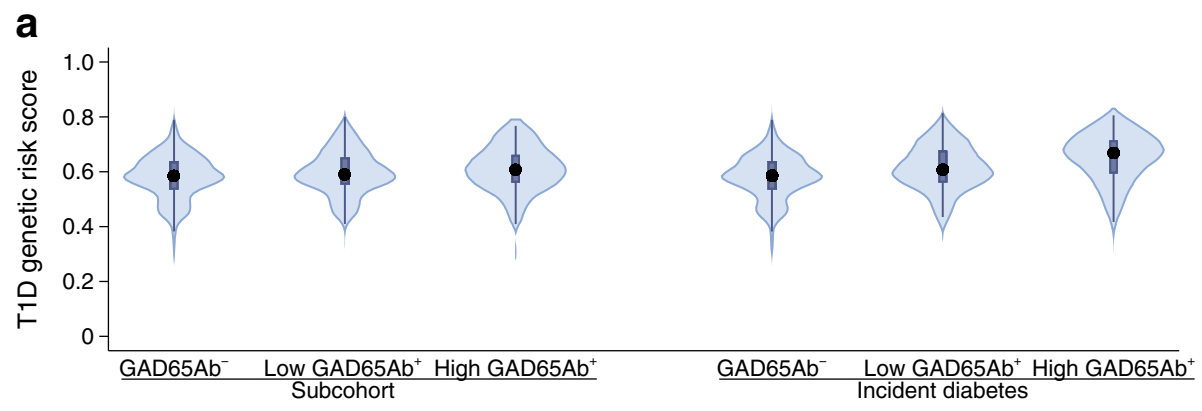

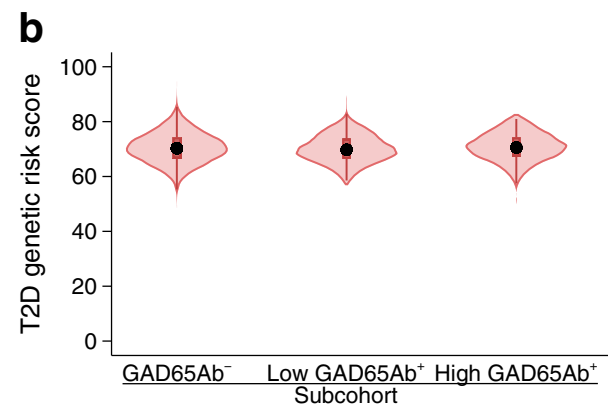

Fig. 1 Violin plots of risk scores according to GAD65 antibody status for (a) type 1 diabetes and (b) type 2 diabetes in the EPIC-InterAct subcohort and incident diabetes cases. $n$ values in (a) are: 12,526 (subcohort, GAD65 $\mathrm{Ab}^{-}$), 149 (subcohort, low GAD65 $\mathrm{Ab}^{+}$), 104 (subcohort, high GAD65 $\mathrm{Ab}^{+}$), 9442 (incident diabetes, GAD65 $\mathrm{Ab}^{-}$), 139 (incident diabetes, low GAD65 $\mathrm{Ab}^{+}$), and 196 (incident diabetes, high GAD65 $\mathrm{Ab}^{+}$). $n$ values in (b) are: 12,249 (subcohort, GAD65 $\mathrm{Ab}^{-}$), 146 (subcohort, low

Among GAD65 antibody-positive individuals there was a significant association of the type 1 diabetes GRS with incident diabetes (HR 2.42 per SD type 1 diabetes GRS; 95\% CI $1.83,3.21)$. There was no evidence of an association among GAD65 antibody-negative individuals (HR 1.00 per SD type 1 diabetes GRS; 95\% CI $0.97,1.04)$. In order to contextualise these interactions, we also analysed the interaction in terms of tertiles of the type 1 diabetes GRS rather than standard deviations. Among the 26,693 individuals with relevant data, $552(2.1 \%)$ were GAD65 antibody positive and $284(1.1 \%)$ were GAD65 antibody positive and also in the top tertile of the type 1 diabetes GRS. Of these 284 individuals, 196 developed incident diabetes and 88 were non-cases. The HR for incident diabetes in the GAD65 antibody-positive individuals comparing the top tertile of the type 1 diabetes GRS with the combination of the other two tertiles was 4.83 (95\% CI 2.47, 9.43). When the exposed group was defined as those who were GAD65 antibody positive and in the top tertile of the type 1 diabetes GRS, the HR for incident diabetes compared with all other individuals was 3.23 (95\% CI 2.10, 4.97). From this it follows that the population attributable fraction, the theoretical fraction of all cases attributable to having GAD65 antibodies and high type 1 diabetes genetic risk, was $1.8 \%$. Baseline characteristics of the subcohort stratified by both GAD65 antibody status and tertile of the type 1 diabetes
GRS (high vs middle/low) are summarised in ESM Table 4 . In a further post hoc analysis, using $\geq 40 \mathrm{U} / \mathrm{ml}$ rather than $\geq 65 \mathrm{U} / \mathrm{ml}$ as the threshold for antibody positivity, the HR for incident diabetes comparing those who were antibody positive and in the top tertile of the type 1 diabetes GRS was 2.05 (95\% CI 1.60, 2.62); the population attributable fraction was $1.7 \%$.

\section{Discussion}

This large, prospective, population-based European study found a significant association between GAD65 antibody positivity and development of incident diabetes after the age of 40 years in individuals free of known diabetes at baseline in eight European countries. A GRS for type 1 diabetes, but not type 2 diabetes, was associated with GAD65 antibody positivity. There was no overall association of the type 1 diabetes GRS with incident diabetes, but, in the subgroup of individuals who were positive for GAD65 antibodies, the type 1 diabetes GRS was strongly associated with incident diabetes. From these data we estimate that just under $2 \%$ of all cases of adult-onset diabetes are attributable to the combination of having a high genetic risk for type 1 diabetes and being positive for GAD65 antibodies. 
Table 2 HRs for incident diabetes comparing GAD65 antibody-positive with GAD65 antibody-negative groups; the EPIC-InterAct study

\begin{tabular}{|c|c|c|c|c|c|c|c|c|c|c|c|c|c|c|c|}
\hline \multirow[t]{2}{*}{ Variable } & \multicolumn{5}{|l|}{ Model 1} & \multicolumn{5}{|l|}{ Model 2} & \multicolumn{5}{|l|}{ Model 3} \\
\hline & $N$ & HR & Lower & Upper & $I^{2}(\%)$ & $N$ & HR & Lower & Upper & $I^{2}(\%)$ & $N$ & HR & Lower & Upper & $I^{2}(\%)$ \\
\hline $\begin{array}{l}\text { GAD65 antibody }{ }^{+} \text {vs } \\
\text { GAD65 antibody }\end{array}$ & $705(+) 26,334(-)$ & 1.80 & 1.48 & 2.20 & 34 & $\begin{array}{l}691(+) \\
25,456(-)\end{array}$ & 1.78 & 1.43 & 2.20 & 36 & $\begin{array}{l}351(+) \\
12,484(-)\end{array}$ & 2.19 & 1.56 & 3.07 & 43 \\
\hline $\begin{array}{l}\text { 'High' GAD65 antibody }{ }^{+} \\
\text {vs GAD65 antibody }\end{array}$ & 353 (high) & 2.46 & 1.91 & 3.17 & 18 & 341 (high) & 2.43 & 1.85 & 3.18 & 19 & 211 (high) & 2.73 & 1.58 & 4.70 & 62 \\
\hline $\begin{array}{l}\text { 'Low' GAD65 antibody }{ }^{+} \\
\text {vs GAD65 antibody }\end{array}$ & 352 (low) & 1.32 & 1.01 & 1.72 & 25 & 350 (low) & 1.28 & 0.99 & 1.66 & 18 & 140 (low) & 1.74 & 1.21 & 2.50 & 3 \\
\hline
\end{tabular}

Model 1: age (as underlying time scale), sex and centre; Model 2: age (as underlying time scale), sex, centre, physical activity, smoking status and education; Model 3: age (as underlying time scale), sex, centre, physical activity, smoking status, education and family history of diabetes

$I^{2}$ represents percentage of variability due to heterogeneity between countries. 'High' GAD65 antibody ${ }^{+}$is defined as GAD65 antibody $\geq 167.5 \mathrm{U} / \mathrm{ml}$ and 'low' GAD65 antibody ${ }^{+}$is defined as GAD65 antibody $<167.5 \mathrm{U} / \mathrm{ml}$

Our study provides definitive evidence of the association between GAD65 antibodies and incident diabetes in European populations, by virtue of the size of the population and extensive follow-up (nearly 4 million person-years), as well as the use of a specific method for defining antibody positivity. An association between higher GAD65 antibody levels and incident diabetes in adults has been suggested previously $[15,16]$. However, the Botnia study was not population-based and was based on relatives of individuals with type 2 diabetes [15], and our previous investigation of adult participants in the Västerbotten Intervention Programme was extremely small and produced estimates of the effect size with wide confidence intervals [16]. A null association between GAD65 antibodies and development of type 2 diabetes reported in Northern Italy may reflect the small size of this study, which was underpowered to detect a true association [18]. The Diabetes Prevention Program also reported no association between GAD65 antibodies and development of type 2 diabetes [19]. However, the follow-up of the Diabetes Prevention Program cohort was very short (3.2 years) and included individuals of various ethnicities with different risks of diabetes, both of which may explain the null overall association. Finally, Sorgjerd et al reported that none of the 349 individuals that developed type 2 diabetes in The Nord-Trøndelag Health Study (HUNT) 3 were positive for GAD65 antibodies in HUNT2 [12]. However, these individuals represented only $23 \%$ of all individuals that developed type 2 diabetes in HUNT3, and type 2 diabetes was classified as absence of GAD65 antibodies. Notably, we did not analyse the GAD65 antibody status at time of diagnosis and assume that a significant portion of these individuals will present GAD65 antibodies at diagnosis. Finally, development of type 2 diabetes in HUNT3 was analysed in a retrospective manner, while our study was conducted prospectively. Therefore, outcomes of these studies are difficult to compare. Our study was restricted to European populations, so we cannot shed any light on ethnic differences in the links between these antibodies and incident diabetes.
Our finding that type 1 diabetes GRS was associated with GAD65 antibody positivity in the subcohort is in line with the study by Mishra et al, which was based on prevalent latent autoimmune diabetes in adults (LADA) cases and nondiabetic control individuals [27]. The HLA types included in our analysis were selected on the basis of their known association with type 1 diabetes as part of an overall type 1 diabetes GRS [35]. The association was mainly explained by the SNPs for HLA-DR3/DR, HLA-DR3/DR4 and HLA-DQB1*0302 (also named HLA-DQ8). We reported in a preliminary smaller study [23] that the presence of HLA-DR3 was associated with GAD65 antibody levels in a non-diabetic population. The same pattern of association for HLA-DR3 was also observed in a study of Finnish school children, where, in addition, HLA-DQB $1 * 0302$ was also associated with GAD65 antibody positivity [21]. However, both of these small studies were cross-sectional and were unable to examine whether the individuals with antibodies and type 1 diabetes risk alleles were more or less likely to develop diabetes later in life.

This study indicates that autoimmunity plays a role in the aetiology of adult-onset diabetes, irrespective of how the people were classified clinically. We estimate that $1.8 \%$ of incident diabetes cases in adulthood are attributable to the combination of having a high genetic risk for type 1 diabetes and having GAD65 antibodies. Our interpretation is that there is an overlap in the aetiology in adult diabetes. It has been shown that presence of GAD65 antibodies predicts future beta cell dysfunction which could be the result of an autoimmune attack on the beta cells (reviewed in [3]), even in people classified as having type 2 diabetes. In some cases, the autoimmune attack does not lead to a total beta cell failure. The result of the autoimmune attack is very dependent on the titres of autoantibodies, presence of multiple antibodies and genetic risk [3]. This notion of a scale of the effect of the autoimmune attack was further strengthened by the finding that transient GAD65 antibodies [12] led to earlier onset of diabetes and contributed to a relative beta cell dysfunction. In line with 
Fig. 2 HRs for incident diabetes comparing GAD65 antibodypositive with GAD65 antibodynegative groups, by country, in the EPIC-InterAct study (Model $2, n=26,147$ )

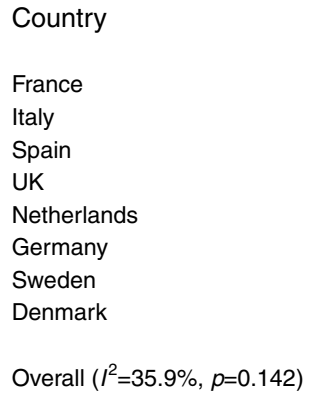

$n$ total $n$ cases

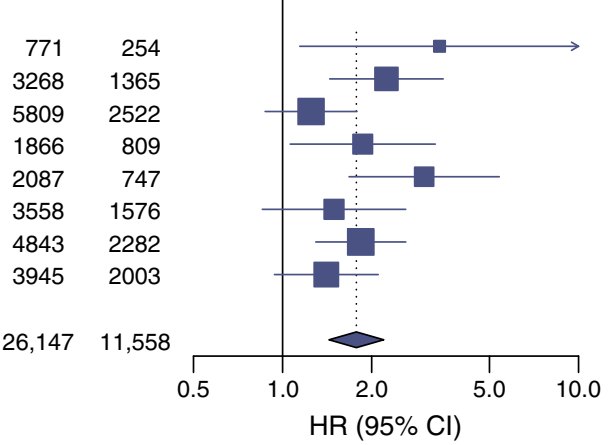

$\mathrm{HR}(95 \% \mathrm{Cl})$

$3.39(1.14,10.06)$

$2.24(1.44,3.49)$

$1.25(0.87,1.78)$

$1.87(1.06,3.28)$

$3.01(1.68,5.40)$

$1.49(0.85,2.61)$

$1.84(1.29,2.61)$

$1.40(0.94,2.10)$

$1.78(1.43,2.20)$ our hypothesis that there is an overlap between aetiologies in adult-onset diabetes, autoimmunity as defined by insulitis assessed by histological investigation was found in pancreases of people diagnosed with type 2 diabetes [36]. However, we can only speculate about the mechanism behind the association, although it has been reported that insulin resistance could be present also among those with GAD65 antibodies [11]. The mechanism could be that insulin resistance increases the secretion of insulin from the beta cells, thereby exposing more antigen (i.e. GAD65, which is co-excreted with insulin from the vesicles in the beta cells) to the immune system. In people with a predisposition for autoimmunity, this autoimmune response towards the beta cells might lead to beta cell dysfunction and/or beta cell death. This hypothesis is supported by findings in an experimental study design [37] that reported an association between GAD65 antibodies and a decrease in maximal insulin secretory capacity in people without diabetes. This suggests that the presence of GAD65 antibodies is a pancreatic marker of a subclinical autoimmune process that could lead to insulin deficiency and subsequently type 2 diabetes.

Taken together, there might be a reason to re-evaluate the present subclassification of diabetes in adulthood [1]. This reevaluation might also lead to studies aimed at the prevention of diabetes, while taking the presence of autoimmunity into account, and to studies trying to optimise the treatment of adult-onset diabetes.
Our study has limitations. The presence of GAD65 antibody at baseline suggests an autoimmune component in the aetiology of adult-onset diabetes over the age of 40 . We deliberately sought to use this descriptive term to avoid forcing individuals into predetermined diagnostic categories, since this has the potential to create circular arguments [38, 39]. We did not have access to GAD65 antibody measurements at the time of diabetes diagnosis but would anticipate that antibodies measured before the diagnosis of diabetes would persist, as previous studies have demonstrated [40, 41]. Several features of our study add to the strength of this analysis. First, all samples were analysed in the same laboratory. The samples were blinded to case or non-case status and samples of participants from different countries were analysed together to minimise assay variation. Second, the cut-off was determined in a subsample of 900 samples, based on specificity of binding, rather than setting an arbitrary cut-off level based on percentiles. Third, the same cut-off level was applied to all samples.

In conclusion, in this large, population-based prospective study, we found that GAD65 antibody positivity was associated with the incidence of diabetes after the age of 40 years. A GRS for type 1 diabetes but not type 2 diabetes was associated with GAD65 antibody positivity and with the incidence of diabetes in those who were GAD65 antibody positive. Our data suggest that incident diabetes in adults includes an element of autoimmune aetiology, which warrants further

Table 3 Associations of type 1 diabetes and type 2 diabetes GRSs with GAD65 antibody positivity in the subcohort and incident diabetes cases; the EPIC-InterAct study

\begin{tabular}{|c|c|c|c|c|c|c|c|c|c|c|}
\hline \multirow[t]{2}{*}{ Variable } & \multicolumn{5}{|c|}{ Subcohort } & \multicolumn{5}{|c|}{ Incident diabetes cases } \\
\hline & $\mathrm{N}$ & OR & Lower & Upper & $\mathrm{I}^{2}(\%)$ & $\mathrm{N}$ & OR & Lower & Upper & $\mathrm{I}^{2}(\%)$ \\
\hline Type 1 diabetes GRS (per $1 \mathrm{SD}$ ) & 12,779 & 1.24 & 1.03 & 1.50 & 46 & 9777 & 1.97 & 1.72 & 2.26 & 12 \\
\hline Type 2 diabetes GRS (per $1 \mathrm{SD}$ ) & 11,054 & 0.97 & 0.85 & 1.12 & 0 & 8228 & 0.85 & 0.76 & 0.96 & 0 \\
\hline
\end{tabular}

Associations are estimated from logistic regression, adjusted for age and sex. Models fit within each country; estimates combined across countries using random effects meta-analysis 
Table 4 HRs (95\% CIs) for incident diabetes comparing GAD65 antibody-positive with GAD65 antibody-negative groups within categories of BMI, WHR (sexspecific tertiles) and tertiles of type 1 diabetes GRS; the EPICInterAct study

\begin{tabular}{llll}
\hline Variable & \multicolumn{2}{l}{ GAD65 antibody ${ }^{+}$vs GAD65 antibody } \\
\cline { 2 - 4 } & $n$ & HR & $95 \%$ CI \\
\hline BMI $\left(\mathrm{kg} / \mathrm{m}^{2}\right)$ & & & \\
$\quad<25$ & 8251 & 3.35 & $(2.51,4.47)$ \\
25 to $<30$ & 10,742 & 1.48 & $(1.14,1.93)$ \\
$\geq 30$ & 6983 & 1.46 & $(1.02,2.08)$ \\
WHR & & & \\
$\quad<0.91$ (men), $<0.77$ (women) & 5170 & 2.91 & $(2.00,4.24)$ \\
0.91 to $<0.96$ (men), 0.77 to $<0.82$ (women) & 6770 & 2.37 & $(1.69,3.30)$ \\
$\geq 0.96($ men), $\geq 0.82$ (women) & 12,364 & 1.33 & $(1.05,1.69)$ \\
Type 1 diabetes GRS & & & $(0.85,1.84)$ \\
$\quad<0.56$ & 6939 & 1.25 & $(0.81,1.60)$ \\
0.56 to $<0.62$ & 7066 & 1.14 & $(1.94,3.29)$ \\
$\geq 0.62$ & 7258 & 2.52 & \\
\hline
\end{tabular}

Models adjusted for age (as underlying timescale), sex, country, physical activity, smoking status and education. Tertiles calculated using distributions in the subcohort prospective studies into the risk factors associated. Moreover, future studies with a different design should investigate the implications of this finding for subclassification of incident diabetes in adults, selection of primary treatment modalities and frequency of risk factor assessment to enhance prognosis.

Acknowledgements We thank all EPIC participants and staff for their contribution to this study. We thank N. Kerrison (MRC Epidemiology Unit, Cambridge, UK) for managing the data and the laboratory team at the MRC Epidemiology Unit, Cambridge for managing the blood samples for the EPIC-InterAct project. We thank the participants of the Spanish EPIC cohort for their contribution to the study as well as the team of trained nurses who participated in the recruitment.

O. Rolandsson: The Västerboten County Council; M. Dorronsoro: We thank the participants of the Spanish EPIC cohort for their contribution to the study as well as to the team of trained nurses who participated in the recruitment; R. Kaaks: German Cancer Aid, German Ministry of Research (BMBF); K. T. Khaw: Medical Research Council UK, Cancer Research UK; T. Kühn: German Cancer Aid, German Cancer Research Center (DKFZ), German Federal Ministry of Education and Research (BMBF); S. Panico: Compagnia di San Paolo; A. M. W. Spijkerman: EPIC Bilthoven and Utrecht acknowledge the Dutch Ministry of Public Health, Welfare and Sports (VWS), Netherlands Cancer Registry (NKR), Statistics Netherlands (the Netherlands); EPIC Ragusa acknowledges for their participation blood donors of AVIS-Ragusa (local blood donors association); Y. T. van der Schouw: EPIC Bilthoven and Utrecht acknowledge the Dutch Ministry of Public Health, Welfare and Sports (VWS), Netherlands Cancer Registry (NKR), Dutch ZON (Zorg Onderzoek Nederland), WCRF, Statistics Netherland; E. Riboli: Imperial College Biomedical Research Centre.

Some of the data were presented as an abstract at the 54th EASD Annual Meeting in 2018.

Data availability The datasets generated during and/or analysed during the current study are available from the corresponding author on reasonable request.

Funding Open access funding provided by Umea University. Funding for the InterAct project was provided by the EU FP6 Programme (grant number LSHM_CT_2006_037197). The autoantibody measurement was funded by Västerbotten County Council and Umeå University, Sweden (OR), the National Institutes of Health (DK26190) (CSH) and the Medical Research Council (MC_UU_12015/1) (NJW). OR: the Västerbotten County Council, Ūmeå University; MDC: Health Research Fund (FIS) of the Spanish Ministry of Health; Murcia Regional Government (N 6236 ); EA: the Health Research Fund (FIS) of the Spanish Ministry of Health and Navarre Regional Government; RK: German Cancer Aid, the German Ministry of Research (BMBF); TJK: Cancer Research UK; KTK: the Medical Research Council UK, Cancer Research UK; PMN: the Swedish Research Council; KO: the Danish Cancer Society; SP: Compagnia di San Paolo; AMWS: the Dutch Ministry of Public Health, Welfare and Sports (VWS), the Netherlands Cancer Registry (NKR), LK Research Funds, Dutch Prevention Funds, Dutch ZON (Zorg Onderzoek Nederland), World Cancer Research Fund (WCRF); Statistics Netherlands; RT: AIREONLUS Ragusa, AVIS-Ragusa, Sicilian Regional Government; AMWS: LK Research Funds, Dutch Prevention Funds, Dutch ZON (Zorg Onderzoek Nederland), World Cancer Research Fund (WCRF); YTvdS: verification of diabetes cases was additionally funded by NL Agency grant IGE05012 and an Incentive Grant from the Board of the UMC Utrecht, LK Research Funds, Dutch Prevention Funds; NGF: MRC core support (MC_UU_12015/5); NIHR Cambridge Biomedical Research Centre (IS-BRC-1215-20014). The funders had no role in study design, data collection and analysis, decision to publish or preparation of the manuscript.

Duality of interest The authors declare that there is no duality of interest associated with this manuscript.

Contribution statement OR, CSH, CL and NJW were responsible for study conception and design and acquisition of data, contributed to statistical analyses and interpretation, drafted the manuscript and obtained funding. SJS performed the statistical analyses and contributed to interpretation and drafting of the manuscript. EA, HB, GF, FRM, PMN, KO, M-DC, MD, MJG, RK, TJK, KTK, VK, TK, DP, SP, CS, M-JS, GS, AMWS, RT, YTvdS, ER and NGF contributed to acquisition of data and reviewed the manuscript. All authors gave final approval of the version to be published. NJW is the guarantor of this work.

Open Access This article is distributed under the terms of the Creative Commons Attribution 4.0 International License (http:// creativecommons.org/licenses/by/4.0/), which permits unrestricted use, 
distribution, and reproduction in any medium, provided you give appropriate credit to the original author(s) and the source, provide a link to the Creative Commons license, and indicate if changes were made.

\section{References}

1. World Health Organization (1999) Definition, diagnosis and classification of diabetes mellitus and its complications: report of a WHO consultation. Department of noncommunicable disease surveillance. WHO, Geneva

2. Arvan P, Pietropaolo M, Ostrov D, Rhodes CJ (2012) Islet autoantigens: structure, function, localization, and regulation. Cold Spring Harb Perspect Med 2:a007658

3. Tuomi T, Santoro N, Caprio S, Cai M, Weng J, Groop L (2013) The many faces of diabetes: a disease with increasing heterogeneity. Lancet 383:1084-1094

4. Zinman B, Kahn SE, Haffner SM, O'Neill MC, Heise MA, Freed MI (2004) Phenotypic characteristics of GAD antibody-positive recently diagnosed patients with type 2 diabetes in North America and Europe. Diabetes 53:3193-3200

5. Hawa MI, Kolb H, Schloot N et al (2013) Adult-onset autoimmune diabetes in Europe is prevalent with a broad clinical phenotype: Action LADA 7. Diabetes care 36:908-913

6. Mollo A, Hernandez M, Marsal JR et al (2013) Latent autoimmune diabetes in adults is perched between type 1 and type 2: evidence from adults in one region of Spain. Diabetes/metabolism research and reviews 29:446-451

7. Zampetti S, Campagna G, Tiberti C et al (2014) High GADA titer increases the risk of insulin requirement in LADA patients: a 7-year follow-up (NIRAD study 7). Eur J Endocrinol 171:697-704

8. Maddaloni E, Lessan N, Al Tikriti A, Buzzetti R, Pozzilli P, Barakat MT (2015) Latent autoimmune diabetes in adults in the United Arab Emirates: clinical features and factors related to insulinrequirement. PloS one 10:e131837

9. Laugesen E, Ostergaard JA, Leslie RD (2015) Latent autoimmune diabetes of the adult: current knowledge and uncertainty. Diabetic medicine 32:843-852

10. Xiang Y, Huang G, Zhu Y et al (2018) Identification of autoimmune type 1 diabetes and multiple organ-specific autoantibodies in adultonset non-insulin-requiring diabetes in China: a population-based multicentre nationwide survey. Diabetes Obes Metab 21:893-902

11. Hjort R, Ahlqvist E, Carlsson PO et al (2018) Overweight, obesity and the risk of LADA: results from a Swedish case-control study and the Norwegian HUNT Study. Diabetologia 61:1333-1343

12. Sorgjerd EP, Asvold BO, Thorsby PM, Grill V (2018) Individuals fulfilling criteria for type 2 diabetes rather than LADA display transient signs of autoimmunity preceding diagnosis with possible clinical implications: the HUNT Study. Diabetes Care 41:e161e163

13. Niu X, Luo S, Li X et al (2019) Identification of a distinct phenotype of elderly latent autoimmune diabetes in adults: LADA China Study 8. Diabetes Metab Res Rev 35:e3068

14. Luk AOY, Lau ESH, Lim C et al (2019) Diabetes-related complications and mortality in patients with young-onset latent autoimmune diabetes: a 14-year analysis of the prospective Hong Kong Diabetes Register. Diabetes Care 42:1042-1050

15. Lundgren VM, Isomaa B, Lyssenko V et al (2010) GAD antibody positivity predicts type 2 diabetes in an adult population. Diabetes 59:416-422

16. Hampe CS, Hall TR, Agren A, Rolandsson O (2007) Longitudinal changes in epitope recognition of autoantibodies against glutamate decarboxylase 65 (GAD65Ab) in prediabetic adults developing diabetes. Clin Exp Immunol 148:72-78

17. Zimmet PZ, Tuomi T, Mackay IR et al (1994) Latent autoimmune diabetes mellitus in adults (LADA): the role of antibodies to glutamic acid decarboxylase in diagnosis and prediction of insulin dependency. Diabet Med 11:299-303

18. Bosi EP, Garancini MP, Poggiali F, Bonifacio E, Gallus G (1999) Low prevalence of islet autoimmunity in adult diabetes and low predictive value of islet autoantibodies in the general adult population of northern Italy. Diabetologia 42:840-844

19. Dabelea D, Ma Y, Knowler WC et al (2014) Diabetes autoantibodies do not predict progression to diabetes in adults: the Diabetes Prevention Program. Diabet Med 31:1064-1068

20. Koopman ADM, Beulens JW, Voerman E et al (2019) The association between GAD65 antibody levels and incident type 2 diabetes mellitus in an adult population: a meta-analysis. Metabolism 95:1-7

21. Knip M, Kukko M, Kulmala P et al (2002) Humoral beta-cell autoimmunity in relation to HLA-defined disease susceptibility in preclinical and clinical type 1 diabetes. Am J Med Genet 115:48-54

22. Rich SS, Akolkar B, Concannon P et al (2009) Overview of the Type I Diabetes Genetics Consortium. Genes Immun 10(Suppl 1): S1-S4

23. Rolandsson O, Hagg E, Janer M et al (2003) High GAD65 autoantibody levels in nondiabetic adults are associated with HLA but not with CTLA-4 or INS VNTR. J Intern Med 253:447-453

24. Bakhtadze E, Cervin C, Lindholm E et al (2008) Common variants in the TCF7L2 gene help to differentiate autoimmune from nonautoimmune diabetes in young (15-34 years) but not in middle-aged (40-59 years) diabetic patients. Diabetologia 51:2224-2232

25. Cervin C, Lyssenko V, Bakhtadze E et al (2008) Genetic similarities between latent autoimmune diabetes in adults, type 1 diabetes, and type 2 diabetes. Diabetes 57:1433-1437

26. Pettersen E, Skorpen F, Kvaloy K, Midthjell K, Grill V (2010) Genetic heterogeneity in latent autoimmune diabetes is linked to various degrees of autoimmune activity: results from the NordTrondelag Health Study. Diabetes 59:302-310

27. Mishra R, Chesi A, Cousminer DL et al (2017) Relative contribution of type 1 and type 2 diabetes loci to the genetic etiology of adult-onset, non-insulin-requiring autoimmune diabetes. BMC Med 15:88

28. Langenberg C, Sharp S, Forouhi NG et al (2011) Design and cohort description of the InterAct Project: an examination of the interaction of genetic and lifestyle factors on the incidence of type 2 diabetes in the EPIC Study. Diabetologia 54:2272-2282

29. Hampe CS, Hammerle LP, Bekris L et al (2000) Recognition of glutamic acid decarboxylase (GAD) by autoantibodies from different GAD antibody-positive phenotypes. J Clin Endocrinol Metab 85:4671-4679

30. Mire-Sluis AR, Gaines Das R, Lernmark A (2000) The World Health Organization International Collaborative Study for islet cell antibodies. Diabetologia 43:1282-1292

31. Rolandsson O, Hagg E, Hampe C et al (1999) Glutamate decarboxylase (GAD65) and tyrosine phosphatase-like protein (IA-2) autoantibodies index in a regional population is related to glucose intolerance and body mass index. Diabetologia 42:555-559

32. The InterAct Consortium (2012) Physical activity reduces the risk of incident type 2 diabetes in general and in abdominally lean and obese men and women: the EPIC-InterAct Study. Diabetologia 55: 1944-1952

33. Oram RA, Patel K, Hill A et al (2016) A type 1 diabetes genetic risk score can aid discrimination between type 1 and type 2 diabetes in young adults. Diabetes Care 39:337-344

34. Morris AP, Voight BF, Teslovich TM et al (2012) Large-scale association analysis provides insights into the genetic architecture and pathophysiology of type 2 diabetes. Nat Genet 44:981-990 
35. Bluestone JA, Herold K, Eisenbarth G (2010) Genetics, pathogenesis and clinical interventions in type 1 diabetes. Nature 464:1293-1300

36. Lundberg M, Seiron P, Ingvast S, Korsgren O, Skog O (2017) Insulitis in human diabetes: a histological evaluation of donor pancreases. Diabetologia 60:346-353

37. Lethagen AL, Ericsson UB, Hallengren B, Groop L, Tuomi T (2002) Glutamic acid decarboxylase antibody positivity is associated with an impaired insulin response to glucose and arginine in nondiabetic patients with autoimmune thyroiditis. J Clin Endocrinol Metab 87:1177-1183

38. Gale EA (2013) Is type 2 diabetes a category error? Lancet 381: 1956-1957

39. Gale EA (2005) Latent autoimmune diabetes in adults: a guide for the perplexed. Diabetologia 48:2195-2199
40. Vermeulen I, Weets I, Costa O et al (2012) An important minority of prediabetic first-degree relatives of type 1 diabetic patients derives from seroconversion to persistent autoantibody positivity after 10 years of age. Diabetologia 55:413-420

41. Decochez K, Keymeulen B, Somers G et al (2000) Use of an islet cell antibody assay to identify type 1 diabetic patients with rapid decrease in C-peptide levels after clinical onset. Belgian Diabetes Registry. Diabetes Care 23:1072-1078

Publisher's note Springer Nature remains neutral with regard to jurisdictional claims in published maps and institutional affiliations.

\section{Affiliations}

Olov Rolandsson ${ }^{1}$ (D) Christiane S. Hampe ${ }^{2} \cdot$ Stephen J. Sharp $^{3} \cdot$ Eva Ardanaz $^{4,5,6} \cdot$ Heiner Boeing $^{7} \cdot$ Guy Fagherazzi $^{8}$. Francesca Romana Mancini ${ }^{8} \cdot$ Peter M. Nilsson $^{9} \cdot$ Kim Overvad $^{10,11} \cdot$ Maria-Dolores Chirlaque $^{5,12}$. Miren Dorronsoro ${ }^{5,13,14}$ - Marc J. Gunter ${ }^{15}$ • Rudolf Kaaks ${ }^{16} \cdot$ Timothy J. Key $^{17} \cdot$ Kay-Tee Khaw $^{18} \cdot$ Vittorio Krogh $^{19}$. Tilman Kühn ${ }^{16}$ • Domenico Palli ${ }^{20}$ • Salvatore Panico ${ }^{21}$ • Carlotta Sacerdote ${ }^{22}$ - Maria-José Sánchez ${ }^{5,23,24}$. Gianluca Severi $^{25,26}$ • Annemieke M. W. Spijkerman ${ }^{27}$ • Rosario Tumino ${ }^{28,29}$ • Yvonne T. van der Schouw ${ }^{30}$. Elio Riboli ${ }^{31} \cdot$ Nita G. Forouhi ${ }^{3} \cdot$ Claudia Langenberg $^{3} \cdot$ Nicholas J. Wareham $^{3}$

1 Department of Public Health and Clinical Medicine, Family Medicine, Umeå University, 90187 Umeå, Sweden

2 Department of Medicine, Division of Metabolism, Endocrinology and Nutrition, University of Washington, Seattle, WA, USA

3 MRC Epidemiology Unit, University of Cambridge School of Clinical Medicine, Institute of Metabolic Science, Cambridge, UK

4 Navarre Public Health Institute, Pamplona, Spain

5 Consortium for Biomedical Research in Epidemiology and Public Health (CIBER Epidemiología y Salud Publica), Madrid, Spain

6 IdiSNA, Navarra Institute for Health Research, Pamplona, Spain

7 Department of Epidemiology, German Institute of Human Nutrition Potsdam-Rehbruecke, Nuthetal, Germany

8 CESP, Faculty of Medicine - University Paris-South, Faculty of Medicine Inserm U1018, University Paris-Saclay, Villejuif, France

9 Department of Clinical Sciences, Clinical Research Center, Skåne University Hospital, Lund University, Malmö, Sweden

10 Department of Public Health, Aarhus University, Aarhus, Denmark

11 Department of Cardiology, Aalborg University Hospital, Aalborg, Denmark

12 Department of Epidemiology, Murcia Regional Health Council, IMIB-Arrixaca, Murcia, Spain

13 Public Health Division of Gipuzkoa, Basque Government, San Sebastian, Spain

14 Instituto BIO-Donostia, Basque Government, San Sebastian, Spain

15 International Agency for Research on Cancer, Lyon, France
16 Division of Cancer Epidemiology, German Cancer Research Center (DKFZ), Heidelberg, Germany

17 Cancer Epidemiology Unit, Nuffield Department of Population Health, University of Oxford, Oxford, UK

18 Department of Public Health and Primary Care, University of Cambridge, Addenbrooke's Hospital, Cambridge, UK

19 Epidemiology and Prevention Unit, Fondazione IRCCS Istituto Nazionale dei Tumori, Milan, Italy

20 Institute for Cancer Research, Prevention and Clinical Network ISPRO, Florence, Italy

21 Dipartimento di Medicina Clinica e Chirurgia, Federico II University, Naples, Italy

22 Unit of Cancer Epidemiology, Azienda Ospedaliera Universitaria (AOU) Citta' della Salute e della Scienza Hospital-University of Turin and Center for Cancer Prevention (CPO), Torino, Italy

23 Andalusian School of Public Health, Granada, Spain

24 Instituto de Investigación Biosanitaria de Granada (ibs.GRANADA), Universidad de Granada, Granada, Spain

25 Inserm, Center for Research in Epidemiology and Population Health (CESP), Université Paris-Sud, Université Paris-Saclay, University of Versailles Saint-Quentin-en-Yvelines (UVSQ) Gustave Roussy, Villejuif, France

26 Facultés de Medicine, Université Paris-Sud, Université ParisSaclay, University of Versailles Saint-Quentin-en-Yvelines (UVSQ) Gustave Roussy, Villejuif, France

27 National Institute for Public Health and the Environment (RIVM), Bilthoven, the Netherlands 
28 Cancer Registry and Histopathology Department, 'Civic - M.P. Arezzo' Hospital, Ragusa, Italy

29 Associazone Iblea per la Ricerca Epidemiologica - Organizazione Non Lucrativa di Utilità Sociale, Ragusa, Italy
30

Julius Center for Health Sciences and Primary Care, University Medical Center Utrecht, Utrecht, the Netherlands

31 School of Public Health, Imperial College London, London, UK 